\title{
Lidil
}

Revue de linguistique et de didactique des langues

$58 \mid 2018$

L'enseignement et l'apprentissage de l'écrit académique à l'aide de corpus numériques

\section{Jean-François de Pietro, Carole Fisher et Roxane Gagnon (dir.), L'oral aujourd'hui : perspectives didactiques}

Namur, Presses universitaires de Namur, collection « Recherches en didactique du français ", $\mathrm{n}^{\circ}$ 9, 2017, $323 \mathrm{p}$.

\section{Anne Sardier}

\section{OpenEdition}

\section{Journals}

Édition électronique

URL : http://journals.openedition.org/lidil/5258

DOI : 10.4000/lidil.5258

ISSN : $1960-6052$

\section{Éditeur}

UGA Éditions/Université Grenoble Alpes

Édition imprimée

ISBN : 978-2-37747-064-8

ISSN : $1146-6480$

\section{Référence électronique}

Anne Sardier, « Jean-François de Pietro, Carole Fisher et Roxane Gagnon (dir.), L'oral aujourd'hui : perspectives didactiques », Lidil [En ligne], 58 | 2018, mis en ligne le 02 novembre 2018, consulté le 25 septembre 2020. URL : http://journals.openedition.org/lidil/5258 ; DOI : https://doi.org/10.4000/lidil. 5258

Ce document a été généré automatiquement le 25 septembre 2020.

(C) Lidil 


\section{Jean-François de Pietro, Carole Fisher et Roxane Gagnon (dir.), L'oral aujourd'hui : perspectives didactiques}

Namur, Presses universitaires de Namur, collection « Recherches en didactique du français ", $n^{\circ}$ 9, 2017, 323 p.

\section{Anne Sardier}

\section{RÉFÉRENCE}

Jean-François de Pietro, Carole Fisher et Roxane Gagnon (dir.), L'oral aujourd'hui : perspectives didactiques, Namur, Presses universitaires de Namur, collection « Recherches en didactique du français ", $\mathrm{n}^{\circ}$ 9, 2017, $323 \mathrm{p}$.

1 L'ouvrage dirigé par Jean-François de Pietro, Carole Fisher et Roxane Gagnon sur la didactique de l'oral, propose des contributions regroupées en quatre parties. Deux contributions constituent la première partie centrée sur deux types de conduites langagières. La première contribution rend compte d'une recherche exploratoire relative aux effets des conduites de justification dans la construction des savoirs grammaticaux d'élèves de 10-11 ans. Les analyses, menées selon un codage minutieux, permettent de distinguer trois types de situations dans lesquelles les élèves s'engagent dans une conduite de justification: pour expliquer, chercher ou débattre. Les auteures recommandent pour finir un "enseignement de l'objet "justification" pour l'apprentissage en grammaire » (p. 63). La deuxième contribution s'intéresse au débat et propose un balayage historique de son enseignement. En distinguant didactique de l'oral / du français, l'auteure note «la profusion des formes» (p. 76) de débats, et, in fine, les possibles invariants propres à cette pratique pour montrer que «l'objet de 
recherche "débat" se formalise au sein des didactiques et répond à leur projet commun et singulier » (p. 80).

2 La deuxième partie de l'ouvrage est composée de cinq contributions centrées sur la construction et l'analyse de dispositifs didactiques. La première contribution questionne les pratiques enseignantes en lien avec les recommandations officielles. Les auteurs notent l'hétérogénéité des pratiques, le fait que «l'oral apparait souvent comme l'oralisation d'un écrit» (p.105) et le manque d'outils pour les enseignants. En réponse, la deuxième contribution propose une "ingénierie didactique » (p. 110) visant la compréhension de l'oral. Les auteurs y exposent leur méthode pour élaborer « des prototypes de séquences didactiques » (p. 114). La contribution suivante porte sur l'exposé. L'auteure y étudie ce qui fait "obstacle» (p.131) à un «enseignement efficace» (p.133) de l'oral. Pour elle, une des principales difficultés concerne les représentations qu'en ont les enseignants. En réponse, la contribution qui suit présente une recherche-action-formation menée au Québec et montre les effets positifs de la mise en place d'« ateliers formatifs» (p.155), notamment sur les représentations et pratiques enseignantes. Pour clore cette partie, une évaluation d'un module d'enseignement dispensé en formation initiale est proposée. Sont questionnées les représentations des étudiants en amont de l'expérience, représentations qui évoluent en cours de travail.

3 La troisième partie regroupe trois propositions relatives aux relations oral/écrit. La première contribution montre judicieusement que les règles relatives à la langue écrite déprécient la langue orale et son locuteur. La deuxième contribution étudie les marques spécifiques de la langue orale dans des productions de lycéens pour " préparer à écrire " (p. 223) et "enseigner à "transposer le parlé" " (p. 228). La contribution suivante donne à voir un oral hétéroclite à travers l'étude de journaux d'apprentissage : l'auteure y distingue "écrit oralisé » chargé de marques propres à l'oral et «oral scripturalisé » (p. 242-243) ; elle y note aussi la présence d'un oral à la fois vecteur d'apprentissage et objet d'enseignement.

4 La dernière partie s'attache aux normes et évaluation de l'oral. Une scrupuleuse étude questionne d'abord la capacité d'autoévaluation de la compétence à communiquer oralement chez de futurs enseignants. Les analyses montrent que «la capacité d'autoévaluation [...] fluctue en fonction des dimensions langagières plutôt que dans le temps » (p. 265) et suggèrent une " confusion des normes » (p. 269) écrites/orales par les étudiants. Après un retour sur le concept de norme(s), la contribution suivante interroge "les représentations de la norme orale du français en contexte FLE/S " (p. 279) chez des apprenants, des enseignants, des linguistes. Des analyses fouillées étudient les variétés d'approches de la norme et reviennent sur l'existence d'un «bon français » (p. 287). La dernière contribution prolonge cette réflexion sur la variation linguistique en s'intéressant aux rétroactions métalangagières évaluatives des enseignants après des prises de parole spontanée des élèves. L'analyse multimodale des discours montre que les normes requises par les enseignants restent relativement aléatoires et parfois hiérarchisées de manière «fantasmée » (p. 306). Dans ce cadre, une « didactique de la variation» (p. 307) semble souhaitable.

5 Finalement, la lecture de cet ouvrage postfacé par Bernard Schneuwly donne à voir l'étendue du champ oral. Quelques points ressortent: le rôle des représentations des enseignants et, en conséquence, la nécessité de mettre d'avantage l'accent sur la formation à l'enseignement-apprentissage par et pour l'oral en classe. 


\section{AUTEURS}

ANNE SARDIER

ACTé, Université Clermont-Auvergne 